\title{
Elementos de motivação e barreiras na utilização de recursos educacionais abertos: um estudo entre uma instituição de ensino superior brasileira e uma alemã
}

Carolina Truzzi da Silva carolina.truzzi@gmail.com 0000-0002-8190-6650 Universidade de São Paulo, São Paulo, São Paulo, Brasil.

Ildeberto Aparecido Rodello rodello@usp.br

0000-0002-8681-355X

Universidade de São Paulo, São Paulo, São Paulo, Brasil.

\section{RESUMO}

Os Recursos Educacionais Abertos têm sido apontados como uma oportunidade de melhoria na qualidade da educação e de compartilhamento de conhecimento e capacitação. Entretanto, existem elementos de motivação para sua utilização e barreiras que dificultam seu acesso. A fim de realizar um estudo comparativo, foram aplicados questionários em duas Instituições de Ensino Superior: uma alemã e outra brasileira, totalizando 76 respondentes. Em relação aos elementos de motivação, identificou-se que as respostas se mantiveram similares em ambas as amostragens, sendo o mais importante "diminuir os custos para os alunos". Em relação às barreiras, no Brasil o fator mais importante foi "falta de acesso à internet", uma barreira da categoria tecnológica, e, na Alemanha, "falta de tempo", uma barreira da categoria social. Assim, conclui-se que as barreiras, por serem externas ao indivíduo, são mais propensas a diferirem em amostragens de características socioeconômicas distintas.
\end{abstract}

PALAVRAS-CHAVE: Recursos educacionais abertos. Educação aberta. Ensino superior. 


\section{INTRODUÇÃO}

Anualmente, a Organização das Nações Unidas (ONU) divulga o ranking dos países pelo seu Índice de Desenvolvimento Humano (IDH) que varia de 0 a 1 e é calculado com base em indicadores de renda, saúde e educação. No relatório divulgado em 2018, a Alemanha estava na quinta posição $(0,936)$ enquanto o Brasil figurava na 79a posição $(0,759)$ do total de 189 países (PROGRAMME, 2018).

A Organisation for Economic Co-operation and Development (OECD, 2018) também realiza estudos comparativos entre países anualmente, porém apenas sobre os 38 países participantes da organização. Nas divulgações mais recentes, no tópico dedicado à educação, a Alemanha figurava na quarta posição do ranking enquanto o Brasil estava na 36a posição, à frente apenas da África do Sul e do México.

Os dados mencionados ilustram o distanciamento socioeconômico entre a Alemanha e o Brasil, o que determina diferenças entre suas estratégias e seus métodos de ensino e as percepções de seus estudantes sobre Educação.

Em 2002, a United Nations Educational, Scientific and Cultural Organization (UNESCO) organizou o Primeiro Fórum Global em Recursos Educacionais Abertos (REA) e formalizou a expressão que hoje se define como "materiais de ensino, aprendizagem e pesquisa em qualquer suporte ou mídia, que estão sob domínio público ou estão licenciados de maneira aberta, permitindo que sejam utilizados ou adaptados por terceiros". A Organização também divulga que acredita que os REA "oferecem uma oportunidade estratégica para melhorar a qualidade da educação, bem como facilitar o diálogo sobre políticas públicas, o compartilhamento de conhecimento e a capacitação" (BUTCHER, 2015)

Dessa forma, os REA podem ser considerados um elemento que pode auxiliar nas estratégias e políticas públicas para a melhoria da qualidade da Educação e também, para a diminuição das desigualdades entre países no que diz respeito à educação. Observa-se que, apesar de o conceito de REA ter sido discutido em 2002, a sua utilização ainda pode ser considerada incipiente nos dias atuais.

Nesse sentido, o artigo apresenta um estudo transversal sobre os elementos de motivação e barreiras na utilização/ reutilização de REA sob a perspectiva dos estudantes de duas Instituições de Ensino Superior (IES): uma alemã e outra brasileira. O objetivo foi identificar os elementos e verificar se há diferenças entre eles principalmente pelo distanciamento socioeconômico entre os dois países. Os resultados podem servir como um indicativo para que estratégias possam ser traçadas com o intuito de ampliar a sua utilização.

A próxima seção engloba o embasamento teórico utilizado para a elaboração deste artigo, com a discussão dos seguintes temas: o conceito "aberto", a importância dos REA, elementos de motivação e barreiras. Em seguida, elucida-se a metodologia utilizada na aplicação da pesquisa e a análise de seus resultados. Então, analisa-se os dados obtidos utilizando métodos estatísticos e, por fim, apresentam-se as conclusões do trabalho. 


\section{RECURSOS EDUCACIONAIS ABERTOS: MOTIVAÇÕES E BARREIRAS}

O conceito "aberto" já foi discutido e definido por diversos autores. Por exemplo, DOWNES (2007) apresenta uma definição como "conveniente, efetivo, financeiramente viável e sustentável e disponível para qualquer estudante e professor no mundo todo". Ainda traz os seguintes critérios para caracterizar um recurso como "aberto": acessível, apropriado, certificado e financeiramente viável.

Segundo Wiley, o termo "aberto" dá a entender que o usuário de REA tem direitos ao trabalhar com o material definidos pelas atividades que compõem os " $4 R$ ". São elas: Reuse (reutilizar - reutilizar o conteúdo sem alterar sua forma), Revise (revisar - adaptar, ajustar, modificar ou alterar o conteúdo), Remix (combinar - combinar conteúdo original ou revisado com outro a fim de gerar algo novo) e Redistribute (compartilhar - compartilhar cópias do conteúdo original, das revisões ou combinações) (WILEY, 2010).

De acordo com Arimoto, Barroca e Barbosa (2015), os REA "vêm abrindo novas possibilidades para a produção e disseminação de conhecimento, ao mesmo tempo em que promovem uma aprendizagem aberta e flexível mais adequada às necessidades individuais" e também "proporcionado oportunidades para inovação em diferentes níveis e modalidades de ensino com significativo impacto sobre a educação".

No Brasil, em função do modelo econômico nacional e da inversão demográfica no século passado, ocorre uma grande concentração da oferta de oportunidades de formação nas grandes metrópoles, causando um desequilíbrio no atendimento educacional nas áreas periféricas ainda mais agravado pelo fraco atendimento global. Assim, os REA surgem também como uma oportunidade de reverter esse cenário, levando Educação à população mais afastada (COSTA; PIMENTEL, 2009).

Figura 1 - Atual Modelo Lógico dos Recursos Educacionais Abertos

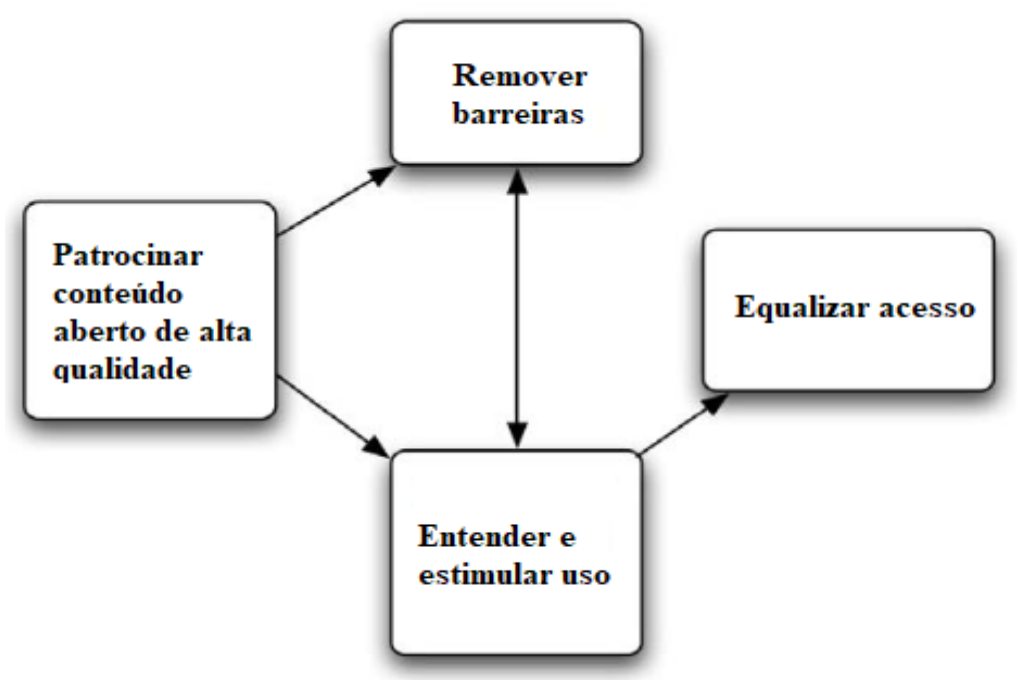

Fonte: Atkins, Brown e Hammond (2007).

A Hewlett Foundation, organização que apoia e patrocina o movimento REA, criou, por meio de um relatório elaborado por Atkins, Brown e Hammond (2007), um modelo lógico com o objetivo de auxiliar na construção de uma comunidade 
para que se incentive um conjunto de stakeholders institucionais a aumentarem e sustentarem essa nova cultura do compartilhamento (Figura 1). Nota-se que, além "patrocinar conteúdo aberto de alta qualidade", os dois termos de importância que compõem o quadro são exatamente "remover barreiras" e "entender e estimular o uso" (ou seja, a motivação), os temas centrais deste estudo.

Bergamini (2003) aponta que "o estudo da motivação humana consiste na pesquisa dos motivos pelos quais as pessoas fazem o que fazem e se encaminham em direção a seus objetivos - objetivos que são, em última análise, escolhas de ordem interior ou intrínsecas à personalidade de cada um." De acordo com Herzberg (1968) e Bergamini (2003), indivíduos possuem fatores que os levam a caminhar em determinada direção, esses fatores podendo ser intrínsecos (internos) ou extrínsecos (externos). Quando são intrínsecos, é chamado de motivação; quando são extrínsecos, se trata de movimento ou satisfação somente.

De acordo com Okada et al. (2013) no contexto de REA, "a motivação é crítica para a criação, a adaptação, a reutilização e, claro, a aprendizagem". Ainda de acordo com a autora, a construção colaborativa do conhecimento é uma oportunidade de aprendizagem orientada por interesses, ritmos e necessidades específicas de cada indivíduo. Assim, a experiência torna-se contextual, resultando na motivação interna, fundamental para todo o processo o uso de diferentes linguagens presentes nos REA, favorecendo o diálogo, interesse e motivação dos estudantes.

Já barreiras, por sua vez, são usualmente entendidas como elementos que impedem a continuação ou desenvolvimento de algo. Em se tratando do uso da tecnologia como um todo, Bedin, Locatelli, Bedin (2016) citam que uma possível barreira encontrada é o próprio professor, que muitas vezes não a insere no processo institucional e que poderia colaborar na aprendizagem dos alunos nos âmbitos culturais, científicos e tecnológicos.

De acordo com Montañola, Oliveira Neto e Rodello (2017), baseado em diferentes autores, as barreiras a REA podem ser classificadas em cinco diferentes categorias:

- Tecnológica: falta de acesso à computadores e internet; problemas com a qualidade da conexão; ausência de softwares apropriados.

- Social: falta de tempo para usar e/ou produzir material compartilhável; falta de incentivos ou recompensas para a criação e uso de REA; ausência de hábito ou interesse por REA; carência de habilidades para desenvolver ou reutilizar REA.

- Legal: desconhecimento sobre sistemas de propriedade intelectual.

- Institucional: falta de políticas e apoio em nível institucional/ nacional/ regional.

- Qualidade: REA com qualidade inferior comparado ao conteúdo tradicional; REA com qualidade duvidosa.

\section{METODOLOGIA DO ESTUDO}

A pesquisa foi realizada com estudantes de duas IES: uma brasileira e outra alemã, escolhidas por conveniência. 0 questionário foi composto por 26 questões 
quantitativas (em duas versões: português e inglês) que abordavam tanto aspectos de caracterização da amostra quanto o conhecimento, a experiência e as percepções dos respondentes em relação a recursos educacionais. Apesar de não possuir relação com a amostragem do projeto, o questionário foi elaborado e validado pelo projeto OER Differentiation (PETE et al., 2018; ROER4D, [S.d.]). Para a aplicação utilizou-se uma amostragem não probabilística por conveniência, totalizando 76 respondentes nos dois países.

Para a análise dos dados, foi realizado um recorte dentro do universo de questões respondidas focalizando nos elementos de motivação e barreiras na utilização de recursos educacionais. Estas questões utilizaram escala do tipo Likert (LIKERT, 1932) para que o respondente sinalizasse o nível de importância/influência percebido em cada uma das alternativas.

Para a questão de elementos de motivação (Quadro 1), as opções de resposta eram "Nada importante" (1), "Sem importância" (2), "Nem importante/nem sem importância" (3), "Importante" (4), "Muito importante" (5) e "Não sei/ não aplicável".

\section{Quadro 1 - Elementos de motivação}

\section{Indique a importância dos seguintes fatores como elementos de motivação para você} usar / reutilizar Recursos Educacionais

Diminuir os custos referentes ao desenvolvimento de cursos para a instituição (Cust_Inst)

Reduzir os custos para os alunos (Cust_Aluno)

É uma prática normal na minha área (Prat_Norm)

Aumenta minha reputação entre os colegas (Reput)

Ajuda outro(s) educador(es)/estudante(s) (Ajud_Edu_Estu)

Sabendo que outros educadores/estudantes podem ver o meu material, pode ajudar a melhorar a qualidade de meu material (Melh_Qual)

Fonte: Autores.

Para a questão das barreiras (Quadro 2), as opções de resposta foram: "Nada" (1), "Um pouco" (2), "Alguma coisa" (3), "Muito" (4), "Extremo" (5) e "Não sei/ Não aplicável". Nos quadros citados também é possível observar a notação (entre parênteses para cada item) que será utilizada nas tabelas ao longo do artigo a fim de facilitar a análise dos resultados obtidos. 
Quadro 2-Barreiras

\begin{tabular}{|}
$\begin{array}{c}\text { Indique até que ponto as seguintes barreiras influenciam o uso de recursos } \\
\text { educacionais }\end{array}$ \\
A falta de acesso à internet (F_Internet) \\
\hline A falta de computador (F_Comput) \\
\hline A Falta de interesse (F__nteresse) \\
\hline A falta de conhecimento acerca de informações sobre a propriedade intelectual do REA \\
(ex: Creative Commons) (F_Conhec) \\
\hline A falta de suporte (F_Suport) \\
\hline A Falta de habilidades pessoais (F_Habil) \\
\hline Eu me preocupo com a qualidade (P_Qualid) \\
\hline A Falta de software (F_Soft) \\
\hline A falta de tempo (F_Tempo) \\
\hline A falta de treinamento (F_Treinam) \\
\hline Não existe compensação (N_Compens) \\
\hline Fão existe recompensa para dedicar tempo e energia para usar recursos educacionais \\
(N_Recomp)
\end{tabular}

\section{APRESENTAÇÃO DOS RESULTADOS}

\section{Caracterização da amostra}

Os 76 estudantes eram de cursos presenciais de Ensino Superior. A amostra brasileira teve 9 participantes do gênero feminino $(21,4 \%)$ e 33 do gênero masculino (78,6\%), com média de idade de 22 anos. Da Alemanha, 29 do gênero feminino $(85,3 \%)$ e 5 do gênero masculino $(14,7 \%)$, com média de idade de 23 anos. Tais dados estão ilustrados no Gráfico 1.

Gráfico 1 - Distribuição de gênero dos respondentes

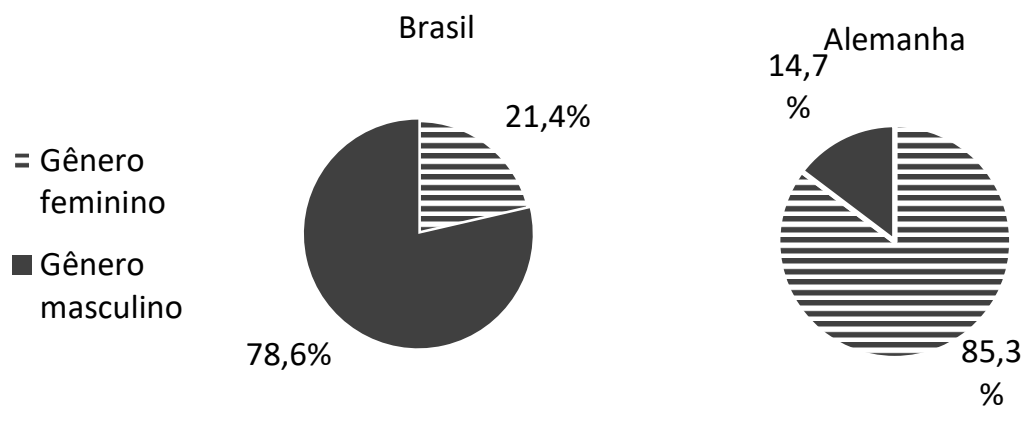

Fonte: Autores. 
A proficiência digital dos respondentes foi dividida em três diferentes estágios, sendo o primeiro "Literácia digital básica - Uso alguns aplicativos comuns de forma eficaz", o segundo "Competência digital intermediária - Posso operar uma gama de aplicativos de forma eficaz" e o terceiro "Especialista digital avançado - Posso explorar aplicações específicas e ferramentas tecnológicas acima de níveis normais". Os resultados podem ser observados no Gráfico 2.

Gráfico 2 - Proficiência digital dos respondentes

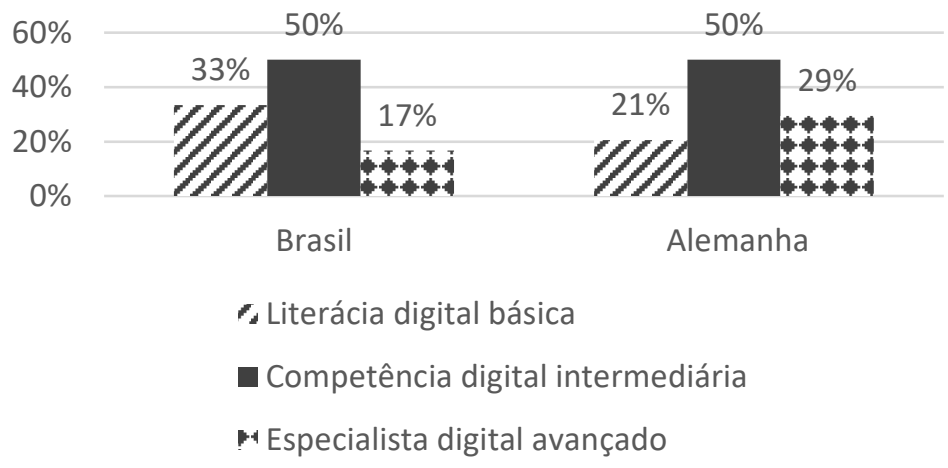

Fonte: Autores.

\section{Fatores de motivação}

Os Gráficos 3 e 4 apresentam os resultados sobre fatores de motivação para as amostras brasileira e alemã, respectivamente. Em ambos os países é possível observar que fator de maior motivação para a utilização ou reutilização de REA é "reduzir os custos para os alunos" (Cust_Alun), no Brasil com 97,6\% das respostas entre "importante" e "muito importante" e, na Alemanha com 73,3\%. Já o segundo fator mais importante diverge entre os países, sendo no Brasil "diminuir os custos referentes ao desenvolvimento de cursos para a instituição" (Cust_Inst), com 81\% das respostas e na Alemanha "ajuda outro(s) educador(es)/ estudante(s)" (Ajud_Edu_Estu) com 64,7\%.

Para os fatores de menor importância, novamente houve uma convergência entre as percepções. A maior concentração de respostas entre "nada importante" e "sem importância" ficaram com as alternativas "sabendo que outros educadores/estudantes podem ver o meu material, pode ajudar a melhorar a qualidade do meu material" (Melh_Qual) (7,2\% - Brasil; 17,6\% - Alemanha) e "aumenta minha reputação entre os colegas" (Reput) (40,5\% - Brasil; 11,7\% Alemanha). 
Gráfico 3 - Distribuição dos resultados sobre elementos de motivação no Brasil

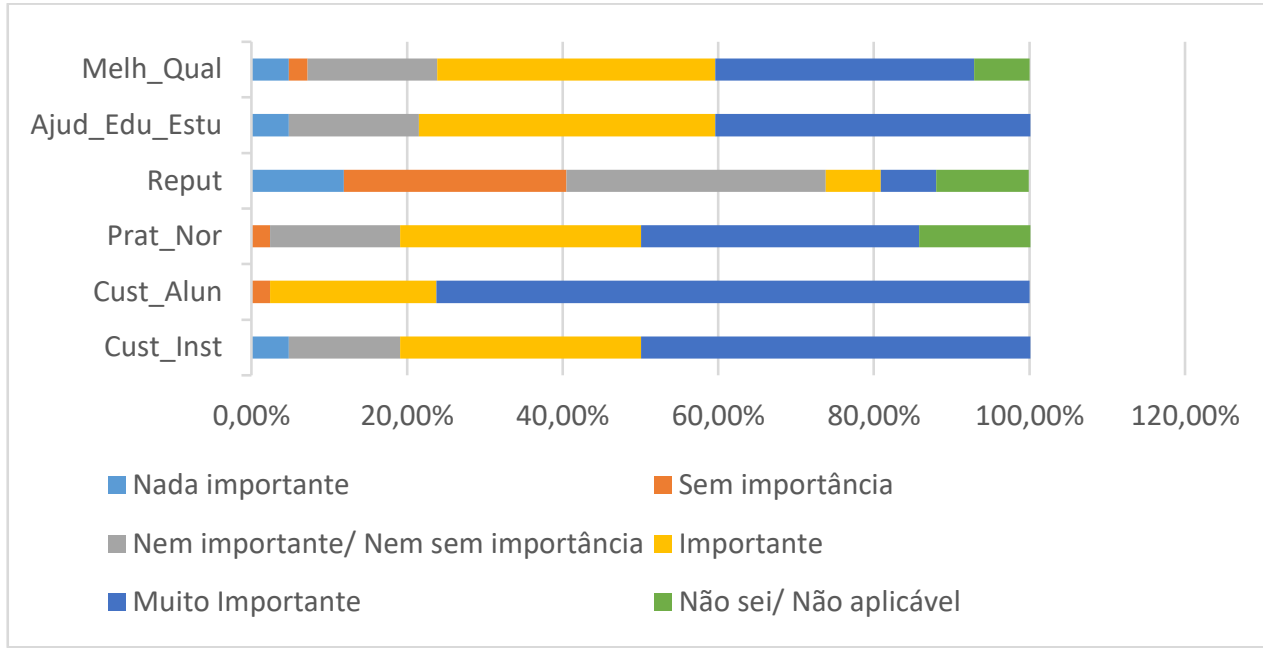

Fonte: Autores.

Gráfico 4 - Distribuição dos resultados sobre elementos de motivação na Alemanha

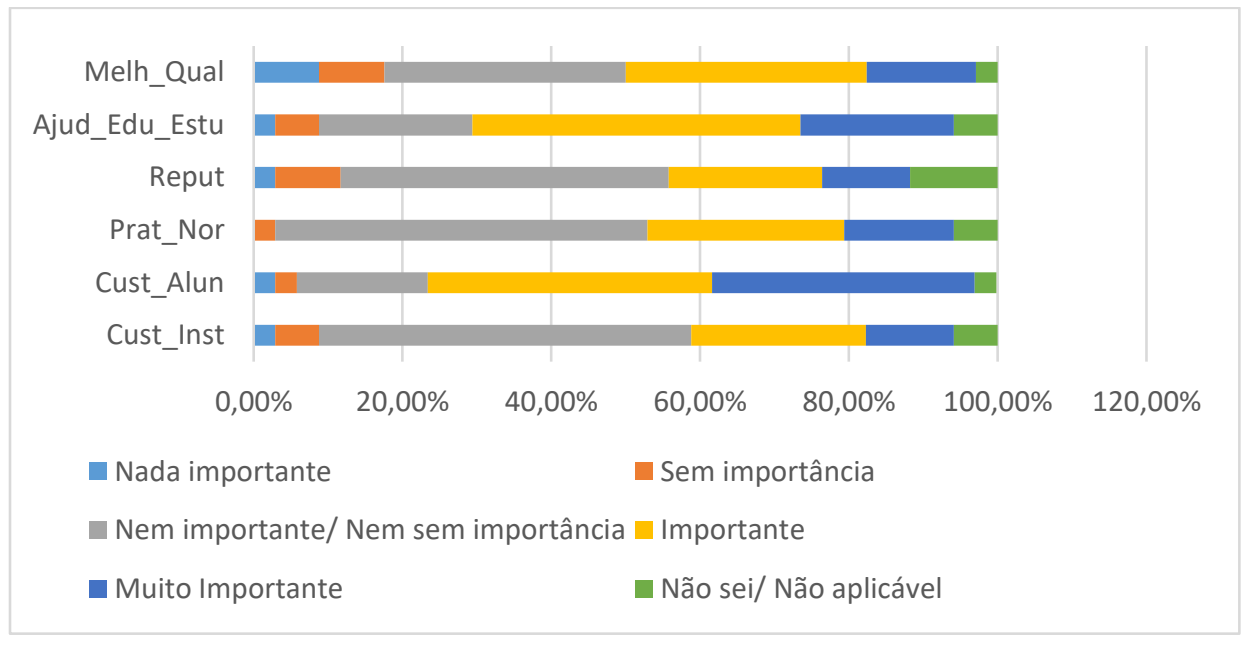

Fonte: Autores.

Nos Gráficos 3 e 4 apresentou-se os dados obtidos no ranqueando da importância das alternativas atribuídas pelos respondentes. Para analisar estatisticamente os dados obtidos pela pesquisa, foi realizado um teste de normalidade de Kolmogorov-Smirnov com correção de Lilliefors com confiabilidade de $95 \%$, em que a significância de todas as alternativas foi menor que 0,05 , ou seja, constatou-se que as amostras não possuem distribuição normal. Assim, decidiu-se aplicar um teste não paramétrico para duas amostras independentes.

Entre os testes possíveis (Qui-quadrado, Mann-Whitney e KolmogorovSmirnov), optou-se pelo teste Mann-Whitney, que melhor se encaixava nas características da amostra. Esse teste permite investigar a hipótese nula de que as médias populacionais são as mesmas para os dois grupos. Cabe ressaltar que, para tal teste, foi considerado nível de confiança de 95\%. Na Tabela 1 é possível observar seus resultados para a questão da motivação. 
Tabela 1 - Teste de Mann-Whitney na questão de Motivação

Hipótese nula

A distribuição da importância de "Diminuir os custos referentes ao desenvolvimento de cursos para a instituição" é igual entre os países

A distribuição da importância de "Reduzir os custos para os alunos" é igual entre os países

A distribuição da importância de "É uma prática normal na minha área" é igual entre os países

A distribuição da importância de "Aumenta minha reputação entre os colegas" é igual entre os países

0,005

0,850

Reter a hipótese nula.
Sig.

\section{Decisão}

Rejeitar a
0,001 hipótese nula.
A distribuição da importância de "Ajuda outro(s) educadore(s)/estudante(s)" é igual entre os países

A distribuição da importância de "Sabendo que outros educadores/estudantes podem ver o meu material, pode ajudar a melhorar a qualidade de meu material" é igual entre os países

\section{Fonte: Autores.}

Das seis hipóteses testadas, foi possível encontrar evidência suficiente para garantir a rejeição de quatro delas:

- "A distribuição da importância de 'Diminuir os custos referentes ao desenvolvimento de cursos para a instituição' é igual entre os países" - nesse caso, mais importante para o Brasil.

- "A distribuição da importância de 'É uma prática normal na minha área' é igual entre os países" - nesse caso, mais importante para a Alemanha.

- "A distribuição da importância de 'Aumenta minha reputação entre os colegas' é igual entre os países" - nesse caso, mais importante para a Alemanha.

- "A distribuição da importância de 'Sabendo que outros educadores/estudantes podem ver o meu material, pode ajudar a melhorar a qualidade de meu material' é igual entre os países" - nesse caso, mais importante para o Brasil.

- Para as demais, não há evidência suficiente para garantir sua rejeição. Isso significa que "reduzir os custos para os alunos" é uma alternativa que obteve distribuição similar nos dois países, ou seja, possuem a mesma importância. Também a alternativa "ajuda outro(s) educador(es)/estudante(s)" teve a hipótese nula retida, possuindo a mesma distribuição/importância nas duas amostras. 


\section{Barreiras percebidas}

O Gráfico 5 apresenta os resultados referentes às barreiras percebidas no uso de REA na amostra brasileira e o Gráfico 6 apresenta os mesmos resultados para a amostra alemã.

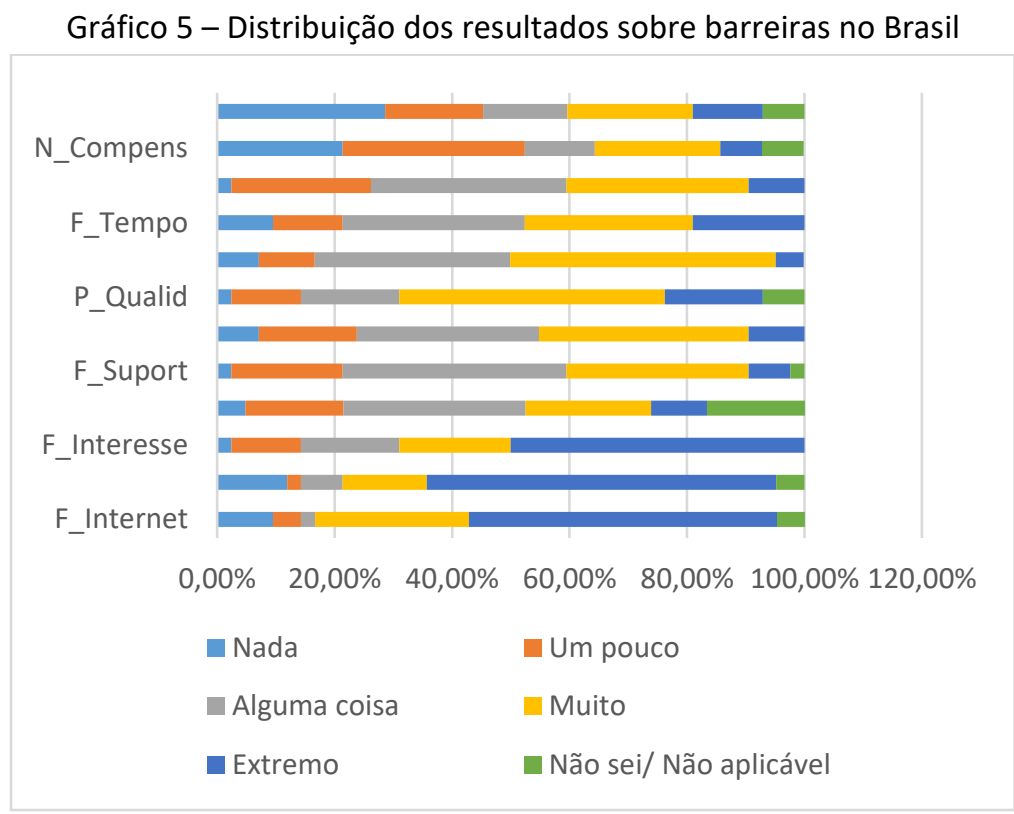

Fonte: Autores.

Em relação às barreiras que mais influenciam no uso de REA, houve menor convergência nos resultados das amostras em comparação às motivações. A barreira de maior importância da amostra brasileira foi "a falta de acesso à internet" (F_Internet) com 78,6\% das respostas e sendo uma barreira da categoria Tecnológica. Na amostra alemã, o item com maior importância atribuída foi "a falta de tempo" (F_Tempo), uma barreira Social, com $58,8 \%$ das respostas.

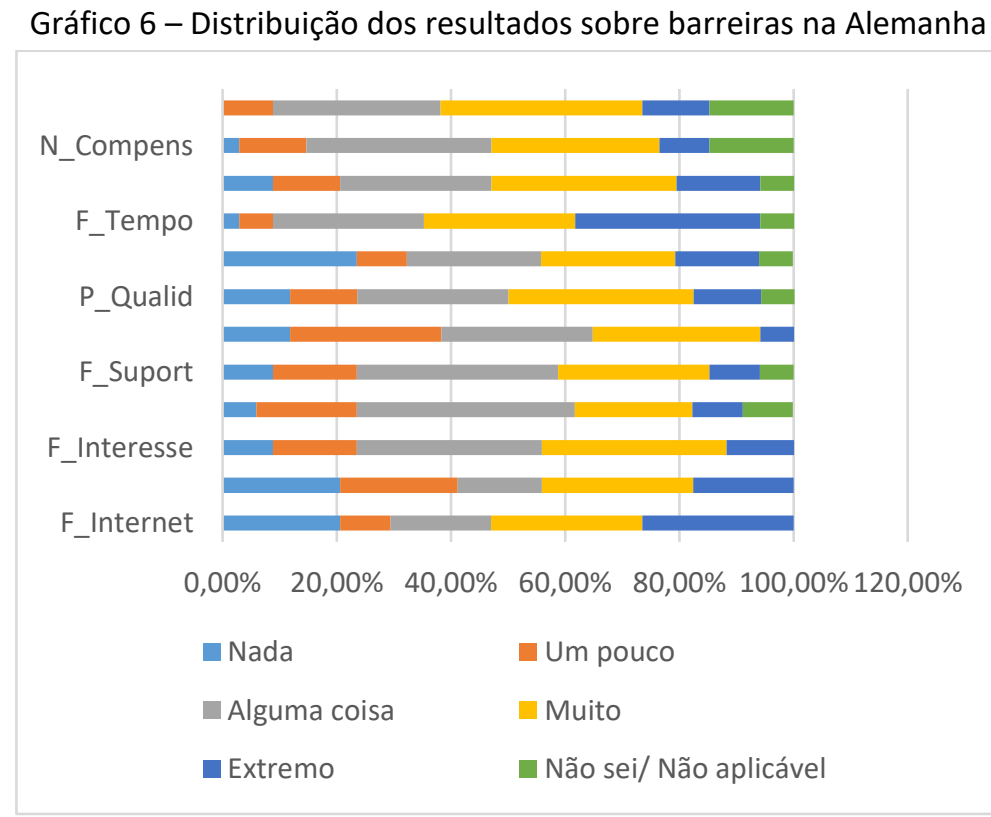

Fonte: Autores. 
Como segunda barreira mais importante, no Brasil obteve-se "a falta de computador" (F_Comput) com 73,4\% das respostas, outra barreira Tecnológica. $\mathrm{Na}$ Alemanha, "a falta de acesso à internet" (F_Internet), com 52,4\% das respostas. É importante ressaltar que esta teve um desvio padrão alto $(1,49)$, em relação aos outros itens da questão, já que $29,9 \%$ das respostas se concentraram nas alternativas de baixa importância.

As barreiras de menor importância para o Brasil foram "não existe compensação" (52,4\%) e "não existe recompensa para dedicar tempo e energia para usar recursos educacionais" (F_Recomp) (45,2\%). Estas barreiras correspondem às categorias Institucional e Social, respectivamente. Já para Alemanha, as barreiras de menor importância foram "falta de computador" (F_Comput) (41,2\%) e "falta de habilidades pessoais" (F_Habil) (38,2\%). Estas barreiras correspondem às categorias Tecnológica e Social, respectivamente.

Para testar estatisticamente, foram seguidos os mesmos procedimentos da questão anterior. Primeiro, testou-se a normalidade da amostra com o teste de normalidade de Kolmogorov-Smirnov com correção de Lilliefors e confiabilidade de $95 \%$. Constatando que a significância de todas as alternativas é menor que 0,05, é possível concluir não se trata de uma amostra com distribuição normal. Da mesma forma, optou-se por realizar o teste Mann-Whitney com nível de confiabilidade de $95 \%$. Na Tabela 2 é possível observar os resultados.

Tabela 2 - Teste de Mann-Whitney para a questão de Barreiras

\begin{tabular}{ccc} 
Hipótese nula & Sig. & Decisão \\
\hline $\begin{array}{c}\text { A distribuição da influência de “A falta de acesso à } \\
\text { internet" é igual entre os países }\end{array}$ & 0,006 & $\begin{array}{c}\text { Rejeitar a } \\
\text { hipótese nula. }\end{array}$ \\
\hline $\begin{array}{c}\text { A distribuição da influência de "A falta de } \\
\text { computador" é igual entre os países }\end{array}$ & 0,000 & $\begin{array}{c}\text { Rejeitar a } \\
\text { hipótese nula. }\end{array}$ \\
\hline $\begin{array}{c}\text { A distribuição da influência de “A Falta de } \\
\text { interesse" é igual entre os países }\end{array}$ & 0,003 & $\begin{array}{c}\text { Rejeitar a } \\
\text { hipótese nula. }\end{array}$
\end{tabular}

A distribuição da influência de " $A$ falta de conhecimento acerca de informações sobre a propriedade intelectual do REA" é igual entre os

0,773

Reter a países

A distribuição da influência de "A falta de suporte" é igual entre os países

0,784

Reter a hipótese nula.

A distribuição da influência de "A Falta de habilidades pessoais" é igual entre os países

0,209

Reter a hipótese nula.

A distribuição da influência de "Eu me preocupo

0,107

Reter a

com a qualidade" é igual entre os países hipótese nula.

A distribuição da influência de "A Falta de software" é igual entre os países

0,339

Reter a hipótese nula. 
A distribuição da influência de "A falta de tempo" é igual entre os países

A distribuição da influência de "A falta de treinamento" é igual entre os países

A distribuição da influência de "Não existe compensação" é igual entre os países

A distribuição da influência de "Não existe recompensa para dedicar tempo e energia para usar recursos educacionais" é igual entre os países

Fonte: Autores.

Das doze hipóteses testadas, foi possível encontrar evidência suficiente para rejeitar quatro delas:

- “A distribuição da influência de 'A falta de acesso à internet' é igual entre os países" - nesse caso, maior para o Brasil.

- “A distribuição da influência de 'A falta de computador' é igual entre os países" - nesse caso, maior para o Brasil.

- “A distribuição da influência de 'A Falta de interesse' é igual entre os países" - nesse caso, maior para o Brasil.

- "A distribuição da influência de 'Não existe recompensa para dedicar tempo e energia para usar recursos educacionais' é igual entre os países" - nesse caso, maior para a Alemanha.

Para as demais, não há evidência suficiente para garantir sua rejeição. Isso significa que a distribuição das respostas pelos estudantes brasileiros e alemães se deu de forma similar nessas alternativas. São elas: "a falta de conhecimento acerca de informações sobre a propriedade intelectual do REA"; "a falta de suporte"; "falta de habilidades pessoais"; "eu me preocupo com a qualidade""; "falta de software", "a falta de tempo" e "a falta de treinamento".

\section{ANÁLISE DOS RESULTADOS}

Ao analisar os resultados sobre os elementos de motivação, percebe-se que, apesar de serem de amostragens de características socioeconômicas distintas, a ordenação da importância atribuída a cada uma das alternativas da pesquisa ocorre de forma similar. Como já citado pelos trabalhos de Herzberg (2008) e Bergamini (2003), a motivação é algo intrínseco, ou seja, mais dificilmente mutável pelo meio em que os indivíduos estão inseridos. Assim, apesar de necessário alguns ajustes de contexto, é possível estudar de forma conjunta estratégias que elucidem aos estudantes os benefícios do uso de REA de acordo com suas motivações.

Em relação às barreiras encontradas, por outro lado, os resultados divergem entre os países, pois barreiras podem ser consideradas como fatores usualmente externos. Nos resultados do Brasil é possível observar que as principais barreiras são da categoria Tecnológica, ou seja, se resumem a dificuldades de infraestrutura, 
como possuir computador ou software adequados para uso de REA. Também no teste não-paramétrico é possível observar que entre as alternativas rejeitadas estão a falta de acesso à internet e de computador, com distribuição divergente da amostra alemã. Como Johnstone (2005) observou em seu trabalho, países em desenvolvimento enfrentam diversas dificuldades no uso de REA, sendo a primeira delas o acesso a uma conexão de internet com velocidade.

Tal resultado corrobora os encontrados pela pesquisa de Montañola, Oliveira Neto e Rodello (2017) sobre barreiras de REA em países sul-americanos, que, além de respondentes brasileiros, contou com respondentes da Colômbia e do Chile. No estudo citado, $80,6 \%$ e $74,6 \%$ dos respondentes atribuíram à falta de acesso à internet e ao computador, respectivamente, importância alta como barreira que influencia o uso de REA. A barreira seguinte também se tratava da característica Tecnológica, com $37,8 \%$ dos respondentes declarando ausência de software como um fator relevante.

Na Alemanha, um país que possui indicadores de desenvolvimento e de Educação mais altos, as questões de infraestrutura não são a maioria absoluta quando considerados os empecilhos atuais, sendo que as barreiras da categoria Tecnológica se tornam menos importantes quando comparadas aos resultados brasileiros (por exemplo, "a falta de acesso à internet": Brasil - 78,3\%; Alemanha - 53\%). Para os estudantes alemães, grande parte das dificuldades são barreiras da categoria Social e, por esse motivo, podem ser combatidas com uma estratégia diferente.

Em uma pesquisa de 2011 da OCDE, a Alemanha declarou que os REA não eram uma questão prioritária para a política educacional alemã. $O$ acesso a materiais digitais não havia sido percebido como um desafio, e a abertura não havia sido percebida como urgente (EHLERS, 2016). De certa forma, essa política alemã talvez justifique algumas das barreiras colocadas pelos estudantes, uma vez que não é uma prioridade para o Governo Alemão.

\section{CONCLUSÃO}

A partir dos resultados apresentados neste estudo, é possível compreender o comportamento e as percepções de estudantes de diferentes características socioeconômicas sobre REA. Tais informações podem ser úteis na elaboração de estratégias e planos de ação para amenizar barreiras e incentivar o seu uso.

$\mathrm{Na}$ questão de motivação, por exemplo, nas duas amostragens, foi identificado que o principal fator de importância para os estudantes que utilizam REA é a diminuição de custos, especialmente por parte dos estudantes. Tal aspecto pode se tornar um fator chave na divulgação dos REA para incentivar seu uso.

Em relação às barreiras, assim como em outros países sul-americanos, percebe-se que a dificuldade do Brasil é relacionada à infraestrutura, podendo ser combatida com maiores investimentos de infraestrutura em suas IES, sendo de recursos públicos ou privados. Apesar de ainda existir algum tipo de dificuldade na Alemanha relacionada ao acesso à internet para uma parcela dos estudantes, a maior parte das barreiras enfrentadas estão relacionadas à rotina dos alunos e outros fatores sociais como a percepção de não haver uma recompensa no uso de REA. Essas questões podem ser combatidas com ajustes na atribuição de notas e da grade horária, por exemplo. 


\title{
ELEMENTS OF MOTIVATION AND BARRIERS IN THE USE OF OPEN EDUCATIONAL RESOURCES: A STUDY BETWEEN A BRAZILIAN AND A GERMAN HIGHER EDUCATION INSTITUTION
}

\begin{abstract}
Open Educational Resources have been identified as an opportunity to improve the quality of education and share knowledge and skills. However, there are elements of motivation for their use and barriers that hinder their access. In order to carry out a comparative study, questionnaires were applied to two Higher Education Institutions: one German and the other Brazilian, totaling 76 respondents. Regarding the elements of motivation, it was identified that the answers remained similar in both samples, being the most important element "to lower the costs for the students". Regarding the barriers, in Brazil the most important factor was "the lack of access to the Internet", a barrier of the technological category, and, in Germany, "lack of time", a barrier of the social category. Thus, it is concluded that the barriers, because they are external to the individual, are more likely to differ in samplings of different socioeconomic characteristics.
\end{abstract}

KEYWORDS: Open educational resources. Open education. Higher education. 


\section{REFERÊNCIAS}

ARIMOTO, Maurício Massaru; BARROCA, Leonor; BARBOSA, Ellen Francine. Recursos Educacionais Abertos: Aspectos de desenvolvimento no cenário brasileiro. Renote, v. 12, n. 2, p. 1-10, 2015. Disponível em: $<$ http://seer.ufrgs.br/index.php/renote/article/view/53458>.

ATKINS, Daniel E; BROWN, John Seely; HAMMOND, Allen L. A Review of the Open Educational Resources (OER) Movement: Achievements, Challenges, and New Opportunities. [S.I: s.n.], 2007. Disponível em: <https://hewlett.org/wpcontent/.../ReviewoftheOERMovement.pdf>.

BEDIN, Everton; LOCATELLI, Aline; BEDIN, Roberto Junior. Tecnologia e Educação: Estado da Arte no paisagismo do SENID. Revista Brasileira de Ensino de Ciência e Tecnologia, v. 9, n. 3, 31 dez. 2016. Disponível em:

$<$ https://periodicos.utfpr.edu.br/rbect/article/view/2744>.

BERGAMINI, Cecília W. Motivação: uma viagem ao centro do conceito. GVexecutivo, v. 1, n. 2, p. 63-67, 2003. Disponível em:

<http://bibliotecadigital.fgv.br/ojs/index.php/gvexecutivo/article/view/34822>.

BUTCHER, Neil. A basic guide to open educational resources (OER). [S.I.]: Commonwealth of Learning (COL);, 2015.

COSTA, Celso José Da; PIMENTEL, Nara Maria. O sistema Universidade Aberta do Brasil na consolidação da oferta de cursos superiores a distância no Brasil. ETD Educação Temática Digital, v. 10, n. 2, p. 71, 7 out. 2009. Disponível em: $<$ https://periodicos.sbu.unicamp.br/ojs/index.php/etd/article/view/978>.

DOWNES, Stephen. Models for Sustainable Open Educational Resources. Interdisciplinary Journal of E-Learning and Learning Objects, v. 3, n. 1, p. 29-44, 2007. Disponivel em: <https://www.learntechlib.org/p/44796>.

EHLERS, Ulf-Daniel. Open Educational Resources in Germany. In: FENGCHUN, MIAO MISHRA, SANJAYA; MCGREAL, RORY (Org.). Open Educational Resources: Policy, Costs and Transformation. France: UNESCO and Commonwealth of Learning, 2016. p. 87-98.

HERZBERG, Frederick. One more time: how do you motivate employees? Boston, Mass: Harvard Business Press, 2008. 
JOHNSTONE, Sally M. Open Educational Resources Serve the World. EDUCAUSE Quarterly, v. 28, n. 3, p. 15-18, 2005. Disponível em: <http://www.educause.edu/EDUCAUSE+Quarterly/EDUCAUSEQuarterlyMagazine Volum/OpenEducationalResourcesServet/157357>.

LIKERT, R. A technique for the measurement of attitudes. Archives of Psychology, v. 22 , n. 140 , p. $1-55,1932$.

MONTAÑOLA, GABRIEL; OLIVEIRA NETO, JOSÉ DUTRA; RODELLO, ILDEBERTO APARECIDO. Barreiras para a utilização de Recursos Educacionais Abertos: um estudo com países sulamericanos. 2017, Ribeirão Preto: [s.n.], 2017.

OECD. OECD Better Life Index for Education. Disponível em: $<$ http://www.oecdbetterlifeindex.org/topics/education/>. Acesso em: 8 jan. 2018.

OKADA, Alexandra. Recursos educacionais abertos e redes sociais. [S.I.]: EDUEMA, 2013.

PETE, Judith et al. Differentiation in Access to, and the Use and Sharing of (Open) Educational Resources among Students and Lecturers at Technical and Comprehensive Ghanaian Universities. Open Praxis, v. 10, n. 4, p. 405-421, 2018.

PROGRAMME, United Nations Development. Human Development Indices and Indicators 2018. [S.I: s.n.], 2018. Disponível em: <https://www.unilibrary.org/content/publication/656a3808-en>.

ROER4D. Research on Open Educational Resources for Development.

WILEY, David. The Open Future. Openness as Catalyst for an Educational Reformation. EducausE review, v. 45, n. 4, p. 15-20, 2010. Disponível em: $<$ http://er.educause.edu/articles/2010/8/openness-as-catalyst-for-aneducational-reformation $>$. 
Recebido: 11 fev. 2019

Aprovado: 25 nov. 2019.

DOI: $10.3895 /$ rbect.v13n2.9547

Como citar: SILVA, C. T.; RODELLO, I. A. Elementos de motivação e barreiras na utilização de recursos educacionais abertos: um estudo entre uma instituição de ensino superior brasileira e uma alemã. Revista

Brasileira de Ensino de Ciência e Tecnologia, Ponta Grossa, v.13, n. 2, p. 61-77, mai./ago. 2020.

Disponível em: <https://periodicos.utfpr.edu.br/rbect/article/view/9547>. Acesso em: XXX.

Correspondência: Ildeberto Aparecido Rodello - rodello@usp.br

Direito autoral: Este artigo está licenciado sob os termos da Licença Creative Commons-Atribuição 4.0

Internacional. 